\title{
Variational Principle Based Stability Analysis of Non-isothermal Film Blowing Process for Non-Newtonian Fluids
}

\author{
Roman Kolarik and Martin Zatloukal \\ Centre of Polymer Systems, Polymer Centre, Tomas Bata University in Zlin, \\ nam. T. G. Masaryka 5555, 76001 Zlin, Czech Republic
}

\begin{abstract}
In this work, a stable numerical scheme for the film blowing stability analysis, considering the variational principle based film blowing model, non-isothermal processing conditions and non-Newtonian behavior of the polymer, has been proposed and used with the aim to reveal the effect of flow activation energy, heat transfer coefficient, mass flow rate and melt temperature on the film blowing stability. It has been found that the model predictions are in good agreement with the corresponding experimental data taken from the open literature.
\end{abstract}

Keywords: Blown film stability, Non-isothermal process, Non-Newtonian fluids, Polymer processing modeling.

PACS: 47.50.-d, 83.10.Gr, 83.50.Uv, 83.60.St, 83.60.Wc, 83.80.Sg

\section{INTRODUCTION}

The beginning of the film blowing process is dated in 1933 where polystyrene was extruding in a tube form in Germany. In 1939, USA applied this technique to polyethylene that became a commercial material [1].

The film blowing process, as one of the oldest processing technologies, is very productive manufacturing process of thin thermoplastic films produced by the film blowing line. In this process, the polymer melt is extruded through an annular die to form a continuous tube which is consequently stretched and inflated by the take-up force and internal bubble pressure until the polymer melt film is fully solidified (see Figure 1). The obtained biaxially oriented polymer films have higher quality physical and optical properties that are applicable in the food processing industry (carrier bags, food wrap film), in the waste industry (garbage bags, waste land fill liners) and in the medical industry (medical films) as well. The typical material of blown film is polyethylene (low-density LDPE, high-density HDPE, linear low-density LLDPE, metallocene $\mathrm{mPE}$ ). Other materials can be polypropylene, polystyrene or polyamide $[2,3]$. 


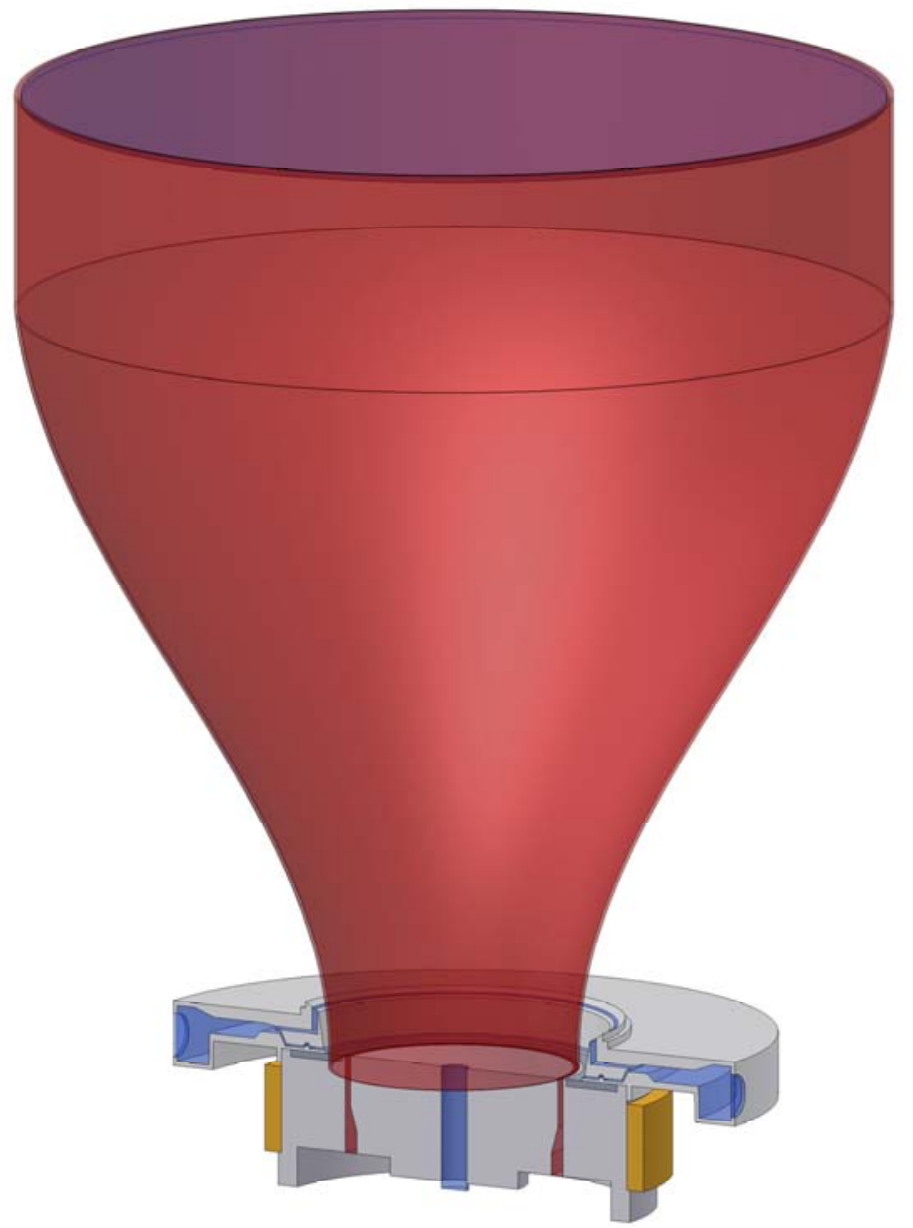

FIGURE 1. Stable bubble formation during the film blowing process.

The main goal of the film blowing process is a production of stable film with good physical and optical properties at a maximum production rate. Nevertheless, the process can be limited by the occurrence of different bubble instabilities such as Draw resonance or Bubble tears [2, 4, 5]. Draw resonance represents periodic diameter oscillation in 2 to 10 -second intervals (see Figure $2 \mathrm{a}$ ) and it occurs at very high strain rates, due to improperly adjusted air ring or during bubble perforation. It has been found that freezeline height increase significantly reduces this type of instability which can be done by reduction of take-up ratio (the ratio of the film velocity above the freezeline height to the melt velocity through die exit), increasing melt temperature or bubble cooling reduction. Stabilization effect can also be achieved by the die design change (narrower die gap) or using higher melt index polymer without strain hardening. Bubble tears instability occurs when the tensile stress at the film exceeds the material strength (see Figure 2b), i.e. when the bubble is drawn too fast or cooled too quickly. This type of instability can be suppressed by cooling rate/take-up ratio reduction, melt temperature increase or die gap narrowing. 

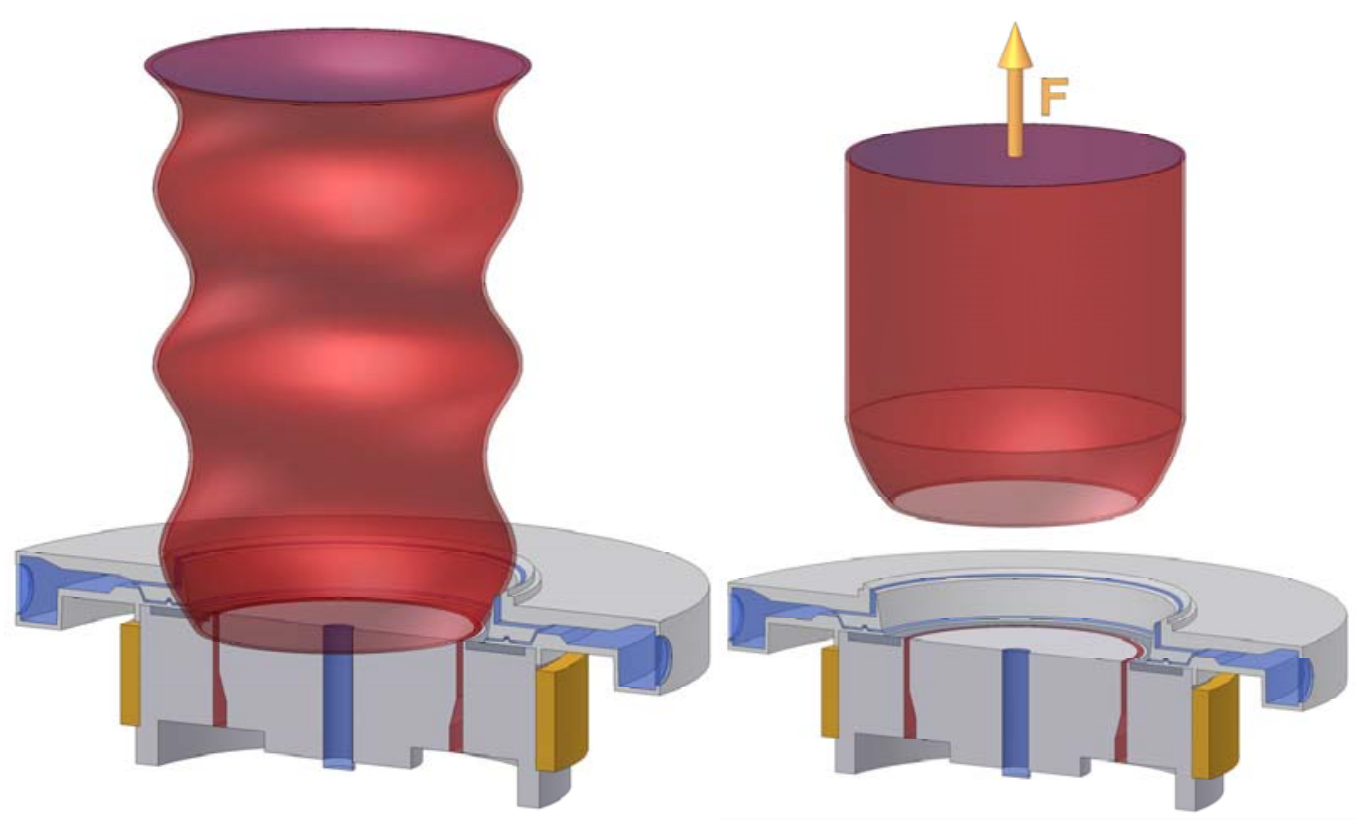

FIGURE 2. Bubble instabilities: (a) Draw resonance (b) Bubble tears.

For better understanding of the conditions at which these unwanted phenomena occur, stability and multiplicity analyses, employing the Pearson-Petrie [6-8] and Cain-Denn [9] formulations, are usually employed. However, it has been shown in [9] that Pearson-Petrie model has limited capability in describing the full range of bubble shapes observed experimentally and, equally important, may lead to variety of numerical instabilities. With the aim to overcome these limitations, stable numerical scheme has been recently developed and applied for the variational principle based Zatloukal-Vlcek film blowing model (where the stable bubble satisfies minimum energy requirements [10]) taking non-isothermal processing conditions and non-Newtonian behavior of the polymer into account [11]. The main aim of this work is to investigate whether this model can also be used for the film blowing stability analysis. For such purpose, the effect of flow activation energy, heat transfer coefficient, mass flow rate and melt temperature on a size of the film blowing stability window will be investigated theoretically and the obtained results will be compared with the experimental data taken from the open literature.

\section{MATHEMATICAL MODELING}

\section{Zatloukal-Vlcek Formulation}

The variational principle based Zatloukal-Vlcek formulation [10] describes a stable film blowing process as a state when the bubble shape satisfies minimum energy requirements; otherwise the process is viewed as unstable. 
The bubble shape is described by the set of simple analytical equations (see Table 1) utilizing four physical parameters: freeze line height, $L$, bubble curvature, $p J$ (which is given by membrane compliance, $J$, and the internal load, $p$ ), the die radius, $R_{0}$ and the blow up ratio, $B U R$ (the ratio of the final bubble diameter at the freezeline height to the bubble diameter at the die exit). It should be mentioned that the equations describing the freezeline height (Eq. 7) and temperature profile (Eq. 8) have been derived in [11] from the cross-sectionally averaged energy equation [12] neglecting the axial conduction, dissipation, radiation effects and crystallization.

TABLE 1. Summary of the Zatloukal-Vlcek model equations.

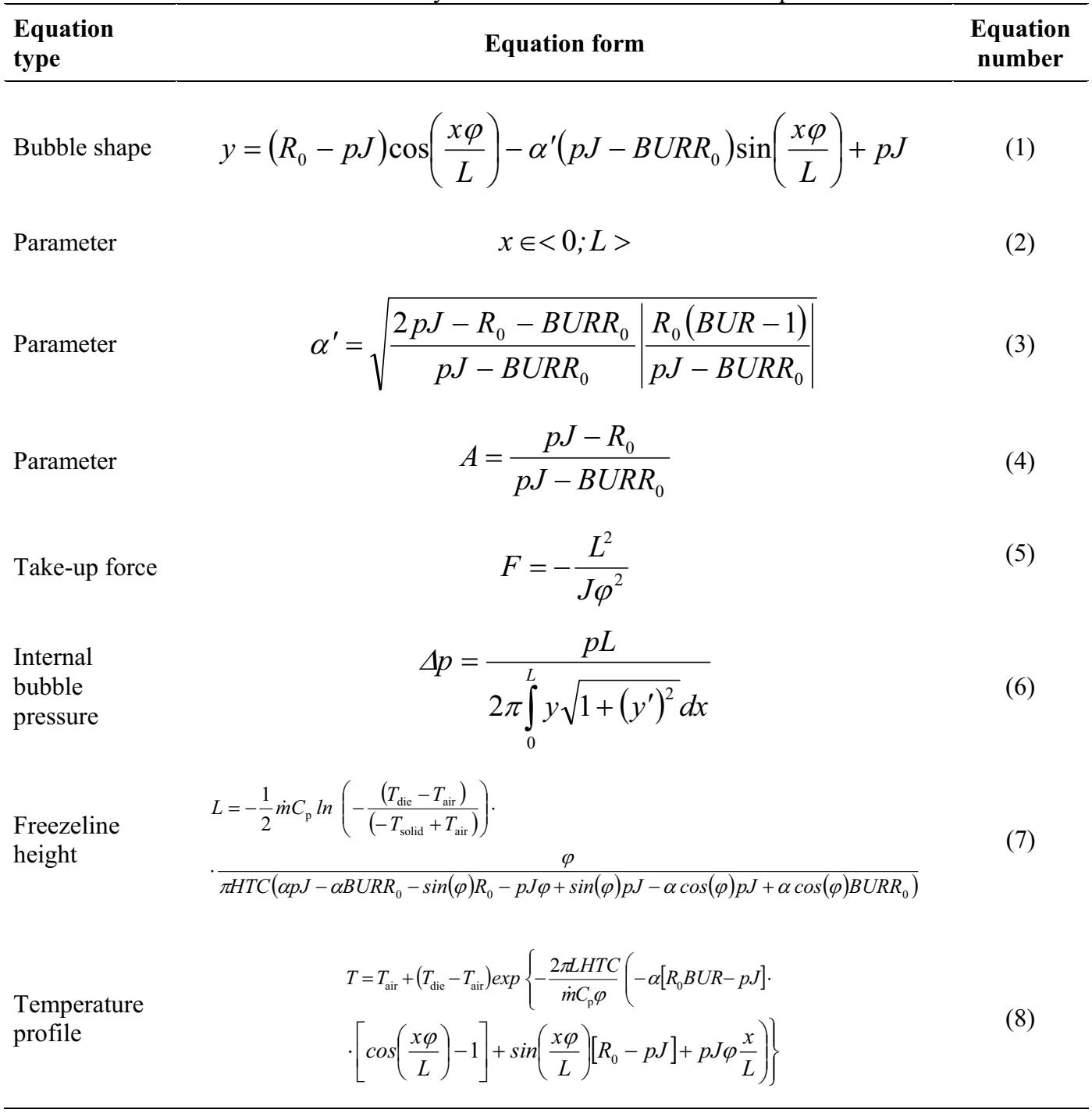

The particular symbols have the following meaning: $C_{\mathrm{p}}$ represents the specific heat capacity, $H T C$ is the heat transfer coefficient, $\dot{m}$ is the mass flow rate, $T_{\text {die }}$ represent the die exit melt temperature, $T_{\text {solid }}$ is the solidification temperature and $T_{\text {air }}$ is the 
cooling air temperature. Parameter $\varphi$ is defined according to Table 2 where a parameter $A$ is defined by Eq. 4.

TABLE 2. Parameters $A$ and $\varphi$ for different bubble shapes (y) (adapted from [13]).

\begin{tabular}{lccc} 
Equation & $\boldsymbol{A}$ & $\boldsymbol{\varphi}$ & $\boldsymbol{y}$ \\
\hline 1. & 1 & 0 & $R_{0}$ \\
2. & $0<A<1$ & $\operatorname{arctg}\left(\frac{\sqrt{1-A^{2}}}{A}\right)$ & The form of Eq. 1 \\
3. & 0 & $\pi / 2$ & $R_{0}\left\{1-\sin \left(\frac{x \pi}{2 L}\right)(1-B U R)\right\}$ \\
4. & $-1<A<0$ & $\pi+\operatorname{arctg}\left(\frac{\sqrt{1-A^{2}}}{A}\right)$ & The form of Eq. 1 \\
5. & -1 & $\pi$ & $\frac{R_{0}}{2}\left\{1+\cos \left(\frac{x \pi}{L}\right)(1-B U R)+B U R\right\}$ \\
\hline
\end{tabular}

\section{Continuity Equation}

$$
Q=2 \pi y(x) h(x) v(x)
$$

In this equation, $Q$ represents the volume flow rate, $y(x)$, the radius of the bubble, $h(x)$, the thickness of the film and $v(x)$ is the film velocity, all as functions of the distance from the die $x$.

\section{Constitutive Equation}

Non-Newtonian behavior of polymer melts is expressed by the constitutive equation derived by generalized Newtonian model which was recently proposed in [14]:

$$
\tau=2 \eta\left(I_{|\mathrm{D}|}, I I_{\mathrm{D}}, I I I_{\mathrm{D}}\right) D
$$

where $\tau$ express the extra stress tensor, $D$ represents the deformation rate tensor and $\eta$ stands for the viscosity which varies with the first invariant of the absolute value of deformation rate tensor $I_{|\mathrm{D}|}=\operatorname{tr}(|D|)$, (where $|D|$ is defined as the square root of $D^{2}$ ) as well as on the second $I I_{\mathrm{D}}=2 \operatorname{tr}\left(D^{2}\right)$, and third, $I I I_{\mathrm{D}}=\operatorname{det}(D)$, invariants of $D$ according to Eq. 11 


$$
\eta\left(I_{|\mathrm{D}|}, I I_{\mathrm{D}}, I I I_{\mathrm{D}}\right)=\eta\left(I I_{\mathrm{D}}\right)^{f\left(I_{|\mathrm{D}|}, I I_{\mathrm{D}}, I I I_{\mathrm{D}}\right)}
$$

where $\eta\left(I I_{\mathrm{D}}\right)$ is given by the well known Carreau-Yasuda model, Eq. 12 and $f\left(I_{|\mathrm{D}|}, I I_{\mathrm{D}}, I I I_{\mathrm{D}}\right)$ is given by Eq. 13

$$
\begin{gathered}
\eta\left(I I_{\mathrm{D}}\right)=\frac{\eta_{0} a_{\mathrm{T}}}{\left[1+\left(\lambda a_{\mathrm{T}} \sqrt{I I_{\mathrm{D}}}\right)^{a}\right]^{\left.\frac{1-n}{a}\right)}} \\
f\left(I_{|\mathrm{D}|}, I I_{\mathrm{D}}, I I I_{\mathrm{D}}\right)=\left\{\tanh \left[\alpha a_{\mathrm{T}}\left(1+\frac{1}{4(\sqrt{3})^{3}}\right)^{-\psi}\left(\left|1+\frac{I I I_{\mathrm{D}}}{I I_{\mathrm{D}}^{3 / 2}}\right|\right)^{\psi} \frac{\sqrt[3]{4\left|I I I_{\mathrm{D}}\right|}+I_{|\mathrm{D}|}}{3}+\beta\right] \frac{1}{\tanh (\beta)}\right\}^{\zeta}
\end{gathered}
$$

In these equations, $\eta_{0}, \lambda, a, n, \alpha, \beta, \zeta$ represent adjustable parameters, whereas parameter $\psi$ is equal to 20 (as suggested in [14]) and $a_{\mathrm{T}}$ is temperature shift factor defined according to the Arrhenius equation:

$$
a_{\mathrm{T}}=\exp \left[\frac{E_{\mathrm{a}}}{R}\left(\frac{1}{273.15+T}-\frac{1}{273.15+T_{\mathrm{r}}}\right)\right]
$$

where $E_{\mathrm{a}}$ is the activation energy, $R$ the universal gas constant, $T_{\mathrm{r}}$ the reference temperature and $T$ is the local bubble temperature.

This recently proposed constitutive equation in [14] has been chosen for the film blowing modeling because it has high flexibility to represent the strain rate dependent steady shear and uniaxial extensional viscosities for linear and branched polyolefines as well as it provides correct behavior in steady planar/equibiaxial extensional viscosity. Moreover, the model allows independent strain hardening level control for planar/equibiaxial extensional viscosity with respect to uniaxial extensional viscosity through parameter $\psi[14]$.

It has been shown in [11] that the equation for the bubble compliance $J$ is defined in the following form:

$$
J=\frac{L^{2} v_{\mathrm{f}}}{2 \varphi^{2} \bar{\eta} \overline{\dot{\varepsilon}_{1}} Q}
$$


where $\bar{\eta}$ and $\bar{\varepsilon}_{1}$ represent the mean values of the melt viscosity $\left(\bar{\eta}=\frac{1}{L} \int_{0}^{L} \eta d x\right)$ and the extensional rate $\left(\overline{\dot{\varepsilon}_{1}}=\frac{1}{L} \int_{0}^{L} \dot{\varepsilon} d x\right)$, respectively, for the whole bubble and $v_{\mathrm{f}}$, is the velocity of the film at the freezeline.

\section{Velocity Profile}

The non-linear velocity profile along the bubble is derived from the force balance in vertical direction (gravity and upward force due to the airflow are neglected) proposed by Pearson and Petrie [6] in the following form:

$$
\frac{2 \pi y h \sigma_{11}}{\sqrt{1+\left(y^{\prime}\right)^{2}}}=F-\pi \Delta p\left(R_{0}^{2} B U R^{2}-y^{2}\right)
$$

where $\sigma_{11}$ is the total stress in the machine direction and $F$ and $\Delta p$ are defined by Eqs. 5 and 6 in Table 1. The deformation rate tensor in the bubble forming region takes the following form:

$$
D=\left(\begin{array}{ccc}
\dot{\varepsilon}_{1} & 0 & 0 \\
0 & \dot{\varepsilon}_{2} & 0 \\
0 & 0 & \dot{\varepsilon}_{3}
\end{array}\right)=\left(\begin{array}{ccc}
\frac{d v}{d x} & 0 & 0 \\
0 & \frac{v}{h} \frac{d h}{d x} & 0 \\
0 & 0 & \frac{v}{y} y^{\prime}
\end{array}\right)
$$

where $v$ and $h$ is bubble velocity and thickness, respectively. Assuming that $h<<y$, then

$$
\sigma_{11}=\tau_{11}-\tau_{22}
$$

By combination of Eqs. 10, 17 and 18, the $\sigma_{11}$ takes the following form:

$$
\sigma_{11}=2 \eta\left(2 \frac{d v}{d x}+\frac{v}{y} y^{\prime}\right)
$$

After substituting Eq. 19 into Eq. 16, the equation for the bubble velocity in the following form can be obtained. 


$$
v=v_{\text {die }} \exp \left(\int_{0}^{L}\left\{\frac{\sqrt{1+\left(y^{\prime}\right)^{2}}\left[F-\pi \Delta p\left(R_{0}^{2} B U R^{2}-y^{2}\right)\right]}{4 Q \eta}-\frac{1}{2 y} y^{\prime}\right\} d x\right)
$$

Having the velocity profile, the deformation rates and the thickness can be properly calculated along the bubble.

\section{Stability Contours Determination}

In this work, the stability processing window is defined as the closed area in the figure at which the relative final film thickness, $H_{1} / H_{0}$, (film thickness at the freezeline height divided by the film thickness at the die) is plotted as the function of the blow up ratio, $B U R$ (see Figure 3 as the example). In this work, the film blowing process is viewed as the unstable process if the process does not satisfy minimum energy requirements [10] $(A<-1$ where $A$ is defined by Eq. 4$)$ or if the film stress in machine or circumference direction reach the rupture stress.

\section{Numerical Scheme}

In the first step, the regular grid of $H_{1} / H_{0}$ versus BUR with equidistant step in both variables is created. For a given grid node, input parameters (see numerical scheme in Figure 4) and guess values for, $\overline{I_{|\mathrm{D}|}}, \overline{I I_{\mathrm{D}}}$ and $\overline{I I I_{\mathrm{D}}}$, so called average bubble viscosity $\bar{\eta}$ can be determined according to Eq. 21

$$
\left.\bar{\eta}=\left\{\frac{\eta_{0} \overline{a_{\mathrm{T}}}}{\left[1+\left(\lambda \overline{a_{\mathrm{T}}} \sqrt{\overline{I I_{\mathrm{D}}}}\right)^{a}\right]\left(\frac{1-n}{a}\right)}\right\}\left\{\tanh \left[\alpha \overline{a_{\mathrm{T}}}\left(1+\frac{1}{4(\sqrt{3})^{3}}\right)^{-\psi}\left(\mid 1+\frac{\overline{I_{\mathrm{D}}}}{\overline{I_{\mathrm{D}}^{3 / 2}}}\right)\right)^{\psi / 3} \frac{\left.\sqrt[3]{4 \overline{I I \mathrm{I}_{\mathrm{D}}} \mid}+\overline{T_{|\mathrm{D}|}}+\beta\right]}{3}\right] \frac{1}{\tanh (\beta)}\right\}^{\zeta}
$$




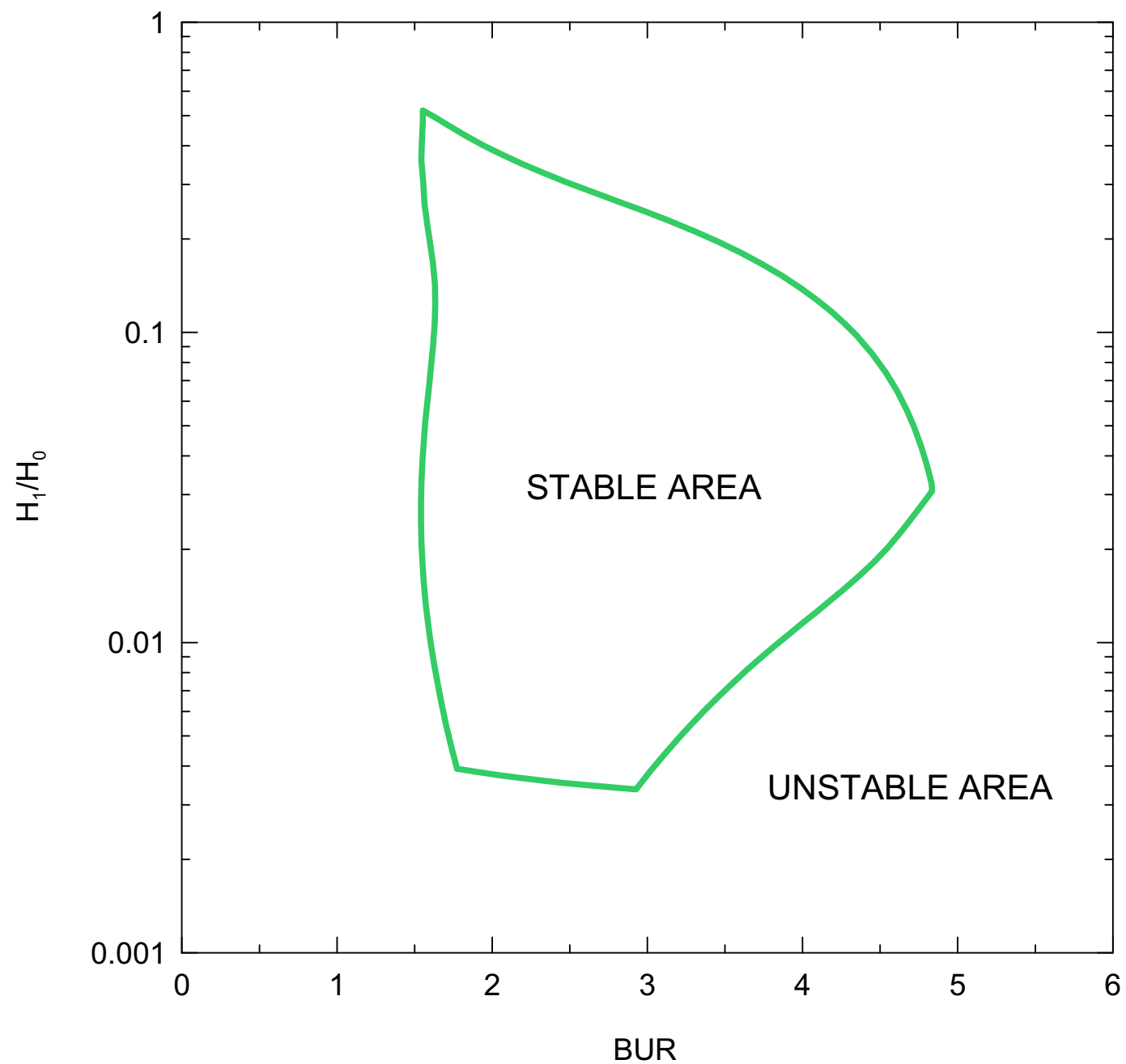

FIGURE 3. Stability area (processing window) predicted by the Zatloukal-Vlcek model.

where $\overline{I_{|\mathrm{D}|}}$ is the mean value of the first invariant of the square root of $D^{2}, \overline{I I_{\mathrm{D}}}$ and $\overline{I I I_{\mathrm{D}}}$ represent the mean value of the second and third invariants of deformation rate tensor, respectively, and $\overline{a_{\mathrm{T}}}$ is the average temperature shift factor:

$$
\begin{gathered}
\overline{I_{|\mathrm{D}|}}=\sqrt{\overline{\dot{\varepsilon}_{1}^{2}}}+\sqrt{\dot{\dot{\varepsilon}}_{2}^{2}}+\sqrt{\dot{\dot{\varepsilon}}_{3}^{2}} \\
\overline{I I_{\mathrm{D}}}=2\left(\overline{\dot{\varepsilon}_{1}^{2}}+\overline{\dot{\varepsilon}_{2}^{2}}+\overline{\dot{\varepsilon}_{3}^{2}}\right) \\
\overline{I I I_{\mathrm{D}}}=\overline{\dot{\varepsilon}_{1}} \overline{\dot{\varepsilon}_{2}} \overline{\dot{\varepsilon}_{3}}
\end{gathered}
$$




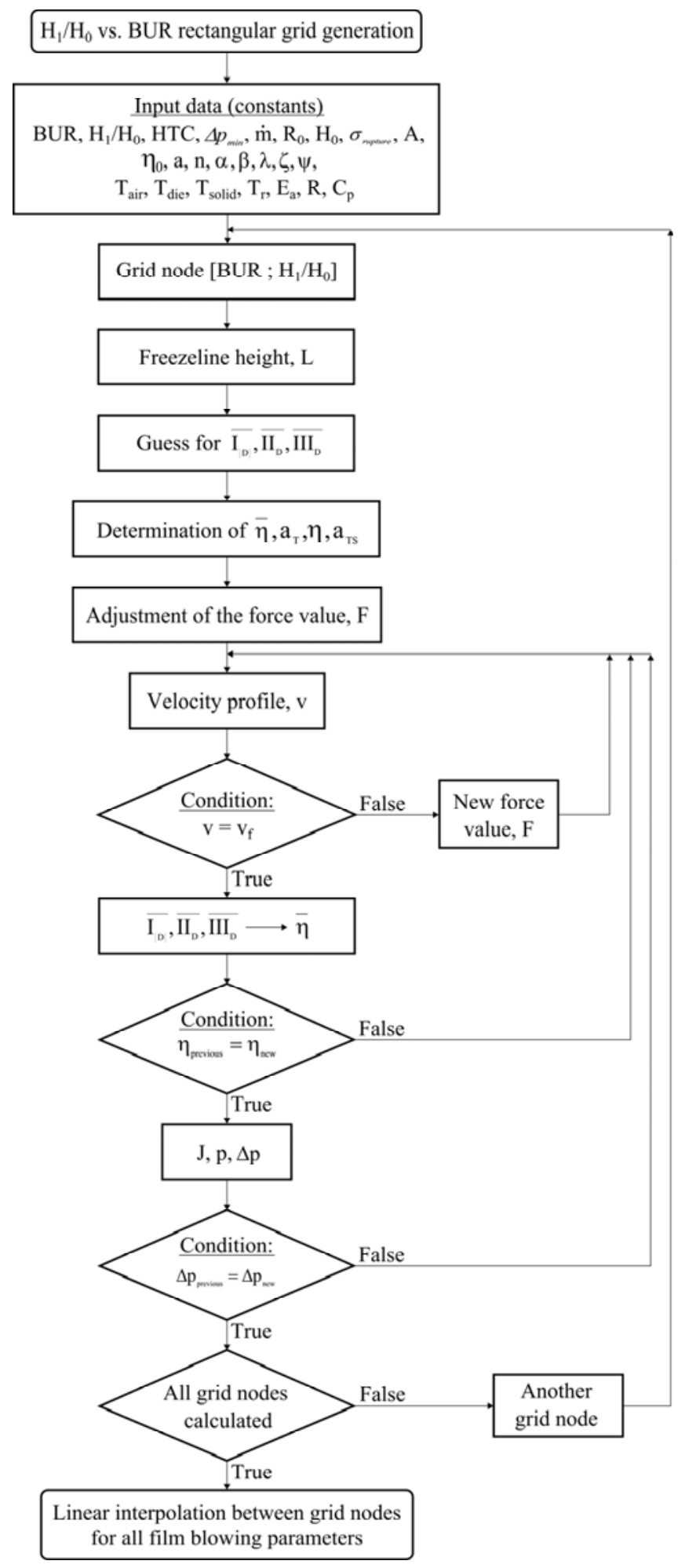

FIGURE 4. Numerical scheme of the Zatloukal-Vlcek model. 


$$
\overline{a_{\mathrm{T}}}=\exp \left[\frac{E_{\mathrm{a}}}{R}\left(\frac{1}{273.15+T_{\mathrm{s}}}-\frac{1}{273.15+T_{\mathrm{r}}}\right)\right]
$$

Here, the average bubble temperature $T_{\mathrm{S}}$ and mean values of the deformation rate components $\overline{\dot{\varepsilon}_{1}}, \overline{\dot{\varepsilon}_{2}}, \overline{\dot{\varepsilon}_{3}}$ are defined as follows

$$
\begin{gathered}
T_{\mathrm{S}}=\frac{T_{\mathrm{die}}+T_{\text {solid }}}{2} \\
\overline{\dot{\varepsilon}_{1}}=\frac{1}{L} \int_{0}^{L} \dot{\varepsilon}_{1} d x \\
\overline{\dot{\varepsilon}_{2}}=\frac{\bar{v}}{\bar{h}} \frac{\bar{h}-H_{0}}{L} \\
\overline{\dot{\varepsilon}_{3}}=-\left(\overline{\dot{\varepsilon}_{1}}+\overline{\dot{\varepsilon}_{2}}\right)
\end{gathered}
$$

where $L$ is freezeline height, $H_{0}$ is bubble thickness at the die, $\bar{v}$ and $\bar{h}$ is mean value of bubble velocity and thickness along the bubble, respectively, which are defined bellow:

$$
\begin{aligned}
& \bar{v}=\frac{1}{L} \int_{0}^{L} v(x) d x \\
& \bar{h}=\frac{1}{L} \int_{0}^{L} h(x) d x
\end{aligned}
$$

It is well known that during the film blowing process, the melt viscosity is changing dramatically between the extrusion die exit and freezeline height. In order to take such strong temperature dependence of the viscosity during velocity calculation of the film (by using Eq. 20) into account, the following expression for the viscosity $\eta$ has been proposed and used:

$$
\eta=\bar{\eta} a_{\mathrm{T}, \text { Bubble }}
$$

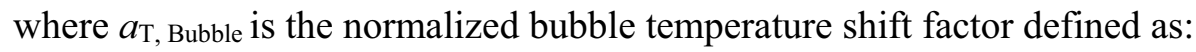

$$
a_{\mathrm{T}, \mathrm{Bubble}}=\frac{a_{\mathrm{T}}}{a_{\mathrm{TS}}}
$$


where $a_{\mathrm{TS}}$ represents the mean value of the Arrhenius temperature shift factor $a_{\mathrm{T}}$ (see Eq. 14) which is given as follows:

$$
a_{\mathrm{TS}}=\frac{1}{L} \int_{0}^{L} a_{\mathrm{T}} d x
$$

The velocity profile is calculated by the help of Eq. 20, where the take-up force $F$ is varied until the calculated film velocity at the freezeline height reached the desirable value (according to defined TUR). For the obtained velocity profile, the average bubble viscosity $\bar{\eta}$ is upgraded (based on the new values of three deformation rate tensor invariants $\overline{I_{|\mathrm{D}|}}, \overline{I I_{\mathrm{D}}}$ and $\overline{I I I_{\mathrm{D}}}$ ) and the velocity calculation is repeated again until the average bubble viscosity $\bar{\eta}$ remains unchanged for the given take-up force $F$ and velocity profile.

Then, the bubble compliance $J$ is calculated according to Eq. 15. For the given $p J$ value (bubble curvature) the internal load, $p$, is determined and consequently used for the internal bubble pressure $\Delta p$ (Eq. 6) calculation. By using this new $\Delta p$ value the velocity profile loop is repeated again until the $\Delta p$ value becomes constant.

The above described procedure is repeated for all $H_{1} / H_{0}$ versus BUR grid nodes. Continues field of given film blowing variable is determined by using the linear interpolation method between each node. The film blowing stability window is then generated on the $H_{1} / H_{0}$ versus BUR mesh for given internal bubble pressure range by all grid nodes for which the stability limit (i.e. for $A=-1$ ) and/or the rupture stress in machine/circumference film direction is reached. Just note that if $\mathrm{A}<-1$ the film blowing process is viewed as unstable because it does not satisfy minimum energy requirements [10].

\section{RESULTS AND DISCUSSION}

The model described in the previous section has been utilized to investigate the effect of flow activation energy, heat transfer coefficient, mass flow rate and melt temperature on the film blowing stability considering reference processing/material conditions, taken from Tas's Ph.D. thesis (experiment no. 23, see Table 3). The Figures 5-12 and Table 4 summarizes the results of the numerical film blowing stability analysis. It also should be mentioned that, stability window wideness is characterized here as its area, which is calculated for each investigated parameter. 
TABLE 3. Film blowing model parameters for Tas's experiment No. 23 [15]

\begin{tabular}{|c|c|c|c|c|c|c|}
\hline \multicolumn{7}{|c|}{ Input parameters for the Zatloukal-Vlcek film blowing model } \\
\hline $\begin{array}{c}H T C \\
\left(\mathbf{W} \cdot \mathbf{m}^{-2} \cdot \mathbf{K}^{-1}\right) \\
\end{array}$ & $\begin{array}{l}\Delta p_{\min } \\
(\mathrm{Pa})\end{array}$ & $\begin{array}{c}\dot{m} \\
\left(\mathrm{~kg}^{\cdot} \mathbf{h}^{-1}\right)\end{array}$ & $\begin{array}{c}R_{0} \\
(\mathbf{m})\end{array}$ & $\begin{array}{c}H_{0} \\
(\mathbf{m}) \\
\end{array}$ & $\begin{array}{l}\sigma_{\text {rupture }} \\
(\mathrm{MPa})\end{array}$ & $\begin{array}{l}A \\
(-)\end{array}$ \\
\hline 52.731 & 85 & 0.00100 & 0.0178 & 0.0022 & 1 & -1 \\
\hline \multicolumn{7}{|c|}{ Parameters of the generalized Newtonian constitutive equation $(\psi=20)$} \\
\hline $\begin{array}{c}\eta_{0} \\
(\mathrm{~Pa} \cdot \mathrm{s})\end{array}$ & $\begin{array}{c}\lambda \\
(\mathbf{s})\end{array}$ & $\begin{array}{c}a \\
(-)\end{array}$ & $\begin{array}{c}n \\
(-)\end{array}$ & $\begin{array}{c}\alpha \\
(s)\end{array}$ & $\begin{array}{c}\beta \\
(-)\end{array}$ & $\stackrel{\zeta}{(-)}$ \\
\hline 2365 & 0.17242 & 0.71597 & 0.37108 & $1 \cdot 10^{-5}$ & $9.21 \cdot 10^{-7}$ & 0.054384 \\
\hline \multicolumn{7}{|c|}{ Temperature parameters } \\
\hline $\begin{array}{l}T_{\text {air }} \\
\left({ }^{\circ} \mathbf{C}\right)\end{array}$ & $\begin{array}{l}T_{\text {solid }} \\
\left({ }^{\circ} \mathrm{C}\right)\end{array}$ & $\begin{array}{c}T_{\text {die }} \\
\left({ }^{\circ} \mathrm{C}\right)\end{array}$ & $\begin{array}{c}T_{\mathrm{r}} \\
\left({ }^{\circ} \mathrm{C}\right)\end{array}$ & $\begin{array}{c}E_{\mathrm{a}} \\
\left(J \cdot \mathrm{mol}^{-1}\right)\end{array}$ & $\begin{array}{c}R \\
\left(J \cdot K^{-1} \cdot \mathrm{mol}^{-1}\right)\end{array}$ & $\begin{array}{c}C_{\mathrm{p}} \\
\left(\mathrm{J}^{\mathrm{kg}} \mathrm{g}^{-1} \cdot \mathrm{K}^{-1}\right)\end{array}$ \\
\hline 25 & 92 & 145 & 190 & 59000 & 8.314 & 2300 \\
\hline
\end{tabular}

TABLE 4. Summary of the calculated data in the Figures 5-12 for the flow activation energy, heat transfer coefficient, mass flow rate and melt/die temperature analysis.

\begin{tabular}{|c|c|c|c|c|c|c|c|c|}
\hline Effect & Point & $\begin{array}{c}B U R \\
(-)\end{array}$ & $\begin{array}{c}H_{1} / H_{0} \\
(-)\end{array}$ & $\begin{array}{c}\Delta p \\
(\mathrm{~Pa})\end{array}$ & $\begin{array}{c}F \\
(\mathbf{N})\end{array}$ & $\begin{array}{c}L \\
(\mathbf{m})\end{array}$ & $\begin{array}{c}\sigma_{11} \\
(\mathbf{M P a})\end{array}$ & $\begin{array}{c}\sigma_{33} \\
(\mathbf{M P a})\end{array}$ \\
\hline \multirow{3}{*}{ 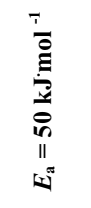 } & $\mathbf{A}$ & 3.049 & 0.002097 & 85 & 5.511 & 0.112276 & 0.867 & 1.000 \\
\hline & B & 2.465 & 0.316228 & 85 & 1.202 & 0.131203 & 0.010 & 0.005 \\
\hline & C & 4.964 & 0.020628 & 513 & 14.780 & 0.076219 & 0.151 & 1.000 \\
\hline \multirow{4}{*}{ 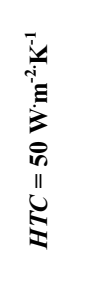 } & $\mathbf{A}$ & 2.490 & 0.003334 & 85 & 6.686 & 0.137370 & 1.000 & 0.514 \\
\hline & B & 1.856 & 0.421697 & 85 & 1.151 & 0.167860 & 0.016 & 0.003 \\
\hline & C & 4.995 & 0.02936 & 725 & 20.343 & 0.079962 & 0.164 & 1.000 \\
\hline & D & 3.018 & 0.003165 & 130 & 7.810 & 0.119327 & 1.000 & 1.000 \\
\hline \multirow{4}{*}{ 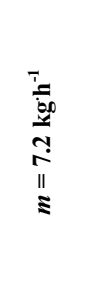 } & $\mathbf{A}$ & 4.254 & 0.003097 & 85 & 10.296 & 0.173026 & 1.000 & 0.945 \\
\hline & B & 4.086 & 0.158489 & 85 & 3.263 & 0.178760 & 0.022 & 0.018 \\
\hline & $\mathrm{C}$ & 6.781 & 0.021774 & 396 & 22.381 & 0.116840 & 0.190 & 1.000 \\
\hline & D & 4.321 & 0.003087 & 88 & 10.446 & 0.170859 & 1.000 & 1.000 \\
\hline \multirow{3}{*}{ 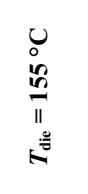 } & $\mathbf{A}$ & 3.209 & 0.002208 & 85 & 4.969 & 0.122842 & 0.937 & 1.000 \\
\hline & B & 2.980 & 0.234374 & 85 & 1.338 & 0.129897 & 0.011 & 0.009 \\
\hline & C & 5.106 & 0.015935 & 585 & 11.979 & 0.084677 & 0.176 & 1.000 \\
\hline
\end{tabular}


Predicted effect of the flow activation energy on the film blowing stability window shape and wideness is presented in Figure 5 and Figure 6, respectively. As can be seen, increase in the flow activation energy leads to widening of the film blowing stability window which is in good agreement with the experimental reality [16] where it has been clearly demonstrated that the film blowing process is more stable for high flow activation energy LDPE in comparison with low activation energy LLDPE polymer. Interestingly, the calculated results depicted in Figure 5 suggest that the relationship between the flow activation energy and minimum achievable film thickness during the film blowing process has no monotonic character.

Predicted effect of the heat transfer coefficient on the film blowing stability window shape and wideness is presented in Figure 7 and Figure 8, respectively. It is clearly visible that the increased bubble cooling intensity (i.e. with the increased heat transfer coefficient) leads to processing window widening and vice versa, which is in good correspondence with the open literature [16]. Moreover, the theoretical results indicate that the high thickness polymer films (which are usually used for the heavy-duty bags production) can only be produced by utilizing highly effective film blowing cooling system.

Predicted effect of the mass flow rate on the film blowing stability window shape and wideness is presented in Figure 9 and Figure 10, respectively. As can be seen, increase of the mass flow rate leads to reduction of the film blowing stability window, which is in agreement with the open literature [16]. The theoretical results depicted in Figure 10 suggest that the effect of the mass flow rate on film blowing stability window width has almost linear character.

Predicted effect of the melt/die temperature on the film blowing stability window shape and wideness is presented in Figure 11 and Figure 12, respectively. As can be seen, increase in the melt/die temperature leads to narrowing of the film blowing stability window, which is in agreement with the open literature [16]. Interestingly, the calculated results depicted in Figure 12 suggest that the relationship between the melt/die temperature and minimum achievable film thickness during the film blowing process has no monotonic character. 


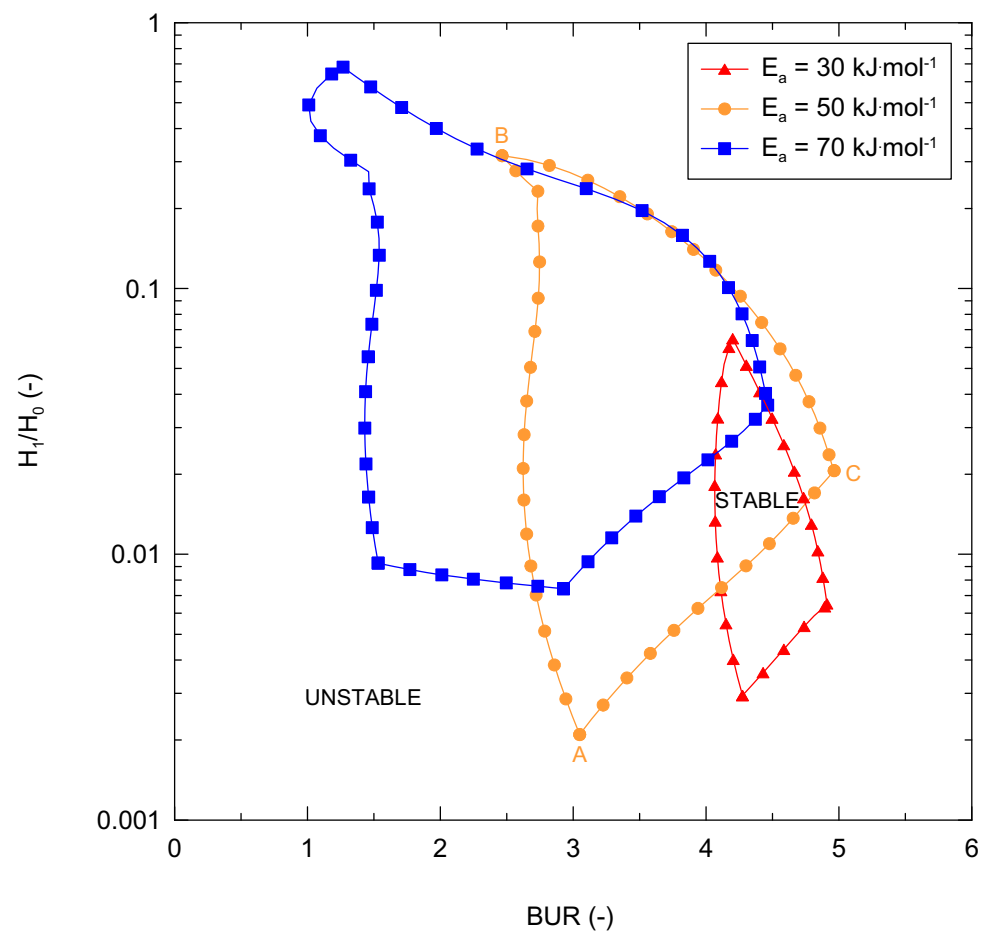

FIGURE 5. Predicted film blowing stability window shape for different level of the flow activation energy.

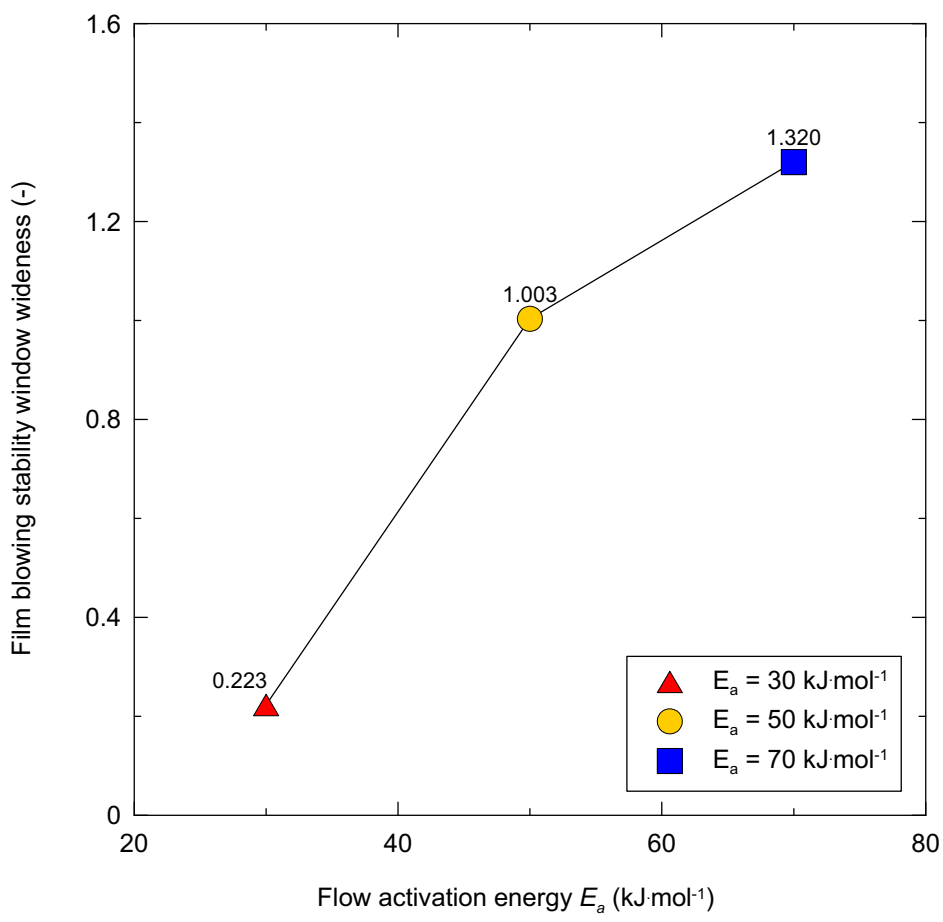

FIGURE 6. Predicted film blowing stability window wideness for different level of the flow activation energy. 


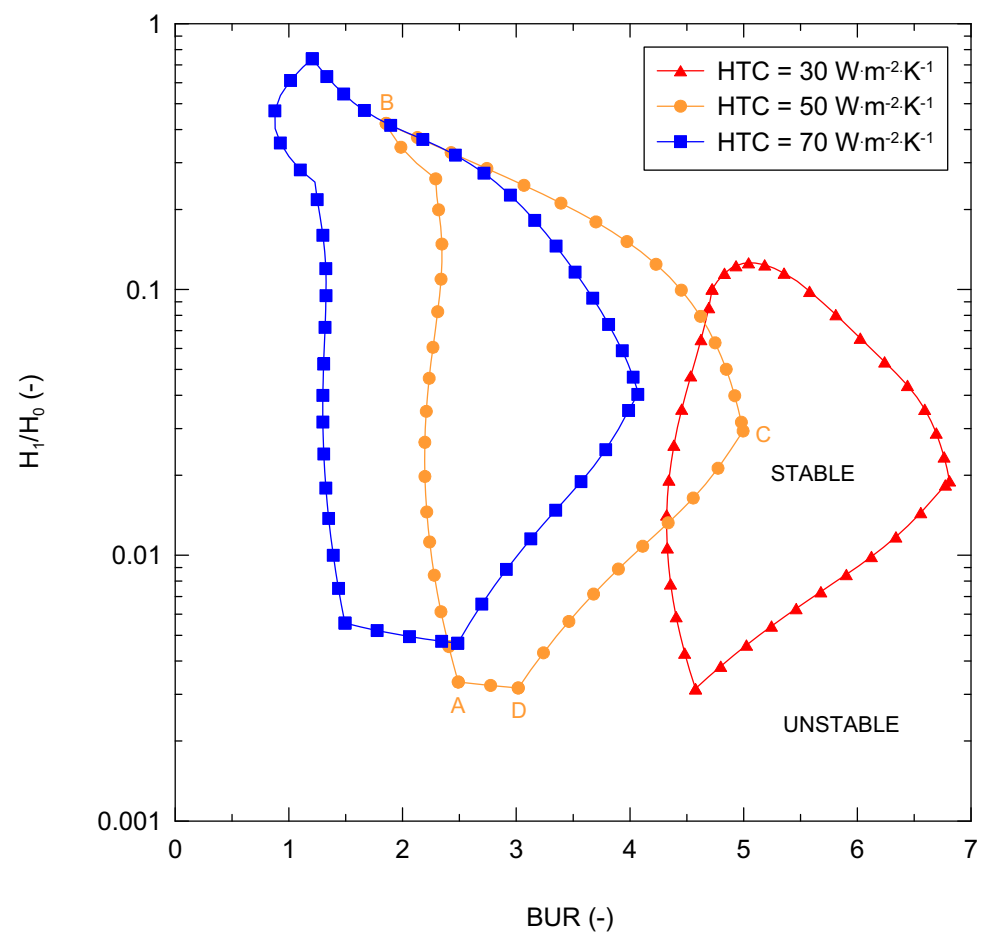

FIGURE 7. Predicted film blowing stability window shape for different level of the heat transfer coefficient.

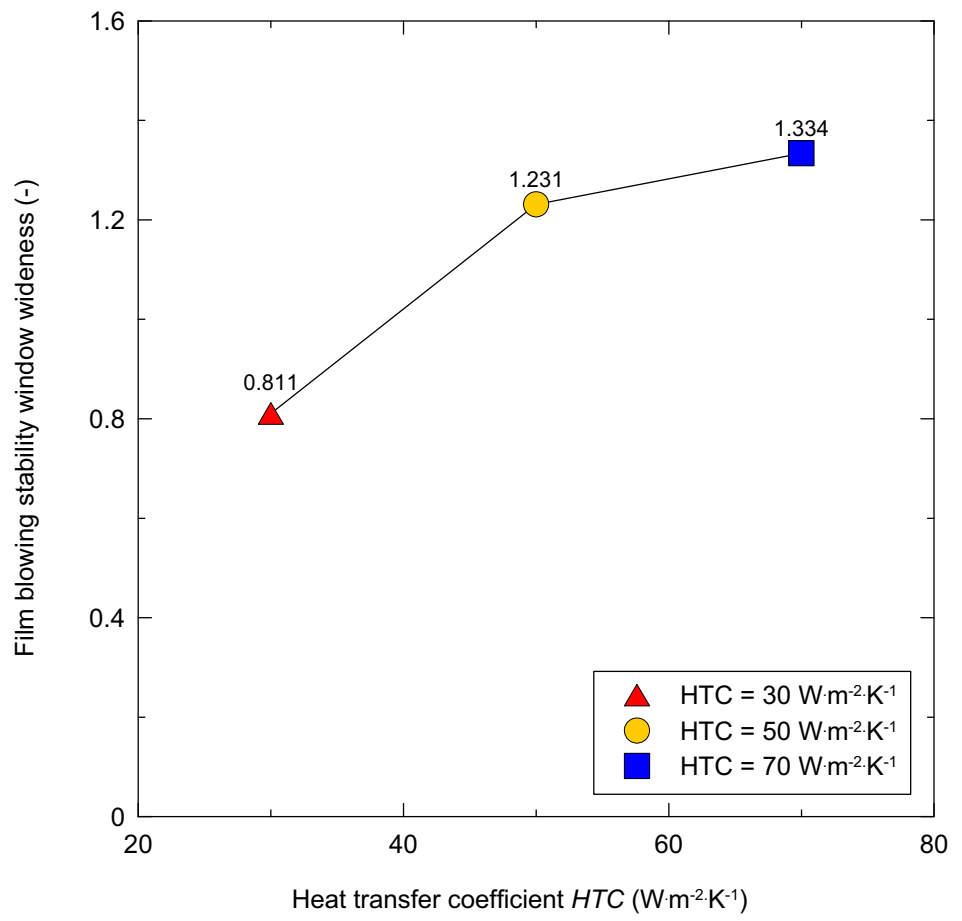

FIGURE 8. Predicted film blowing stability window wideness for different level of the heat transfer coefficient. 


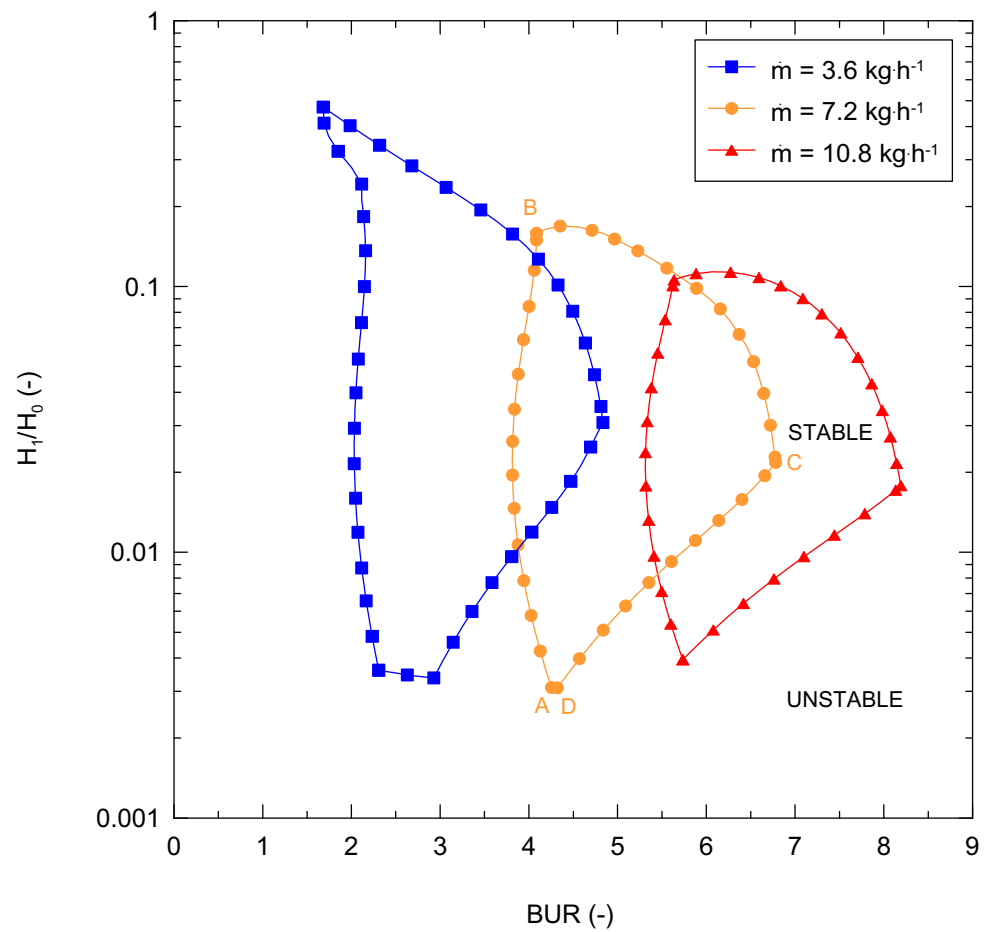

FIGURE 9. Predicted film blowing stability window shape for different level of the mass flow rate.

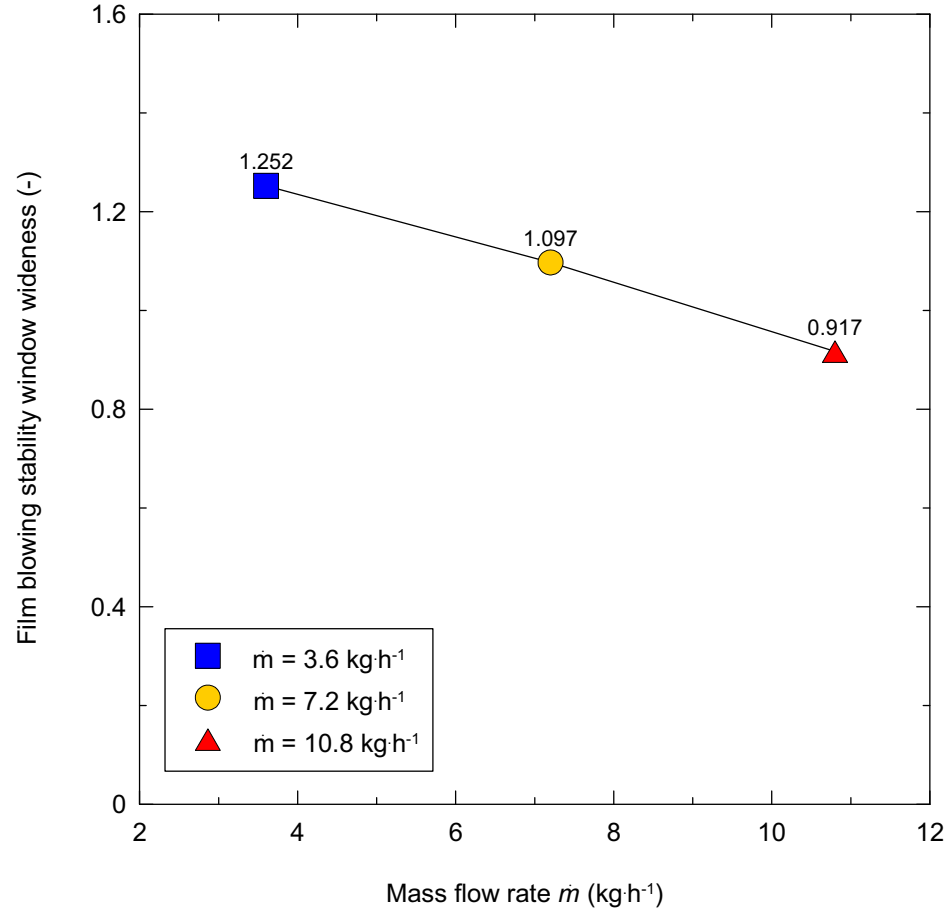

FIGURE 10. Predicted film blowing stability window wideness for different level of the mass flow rate. 


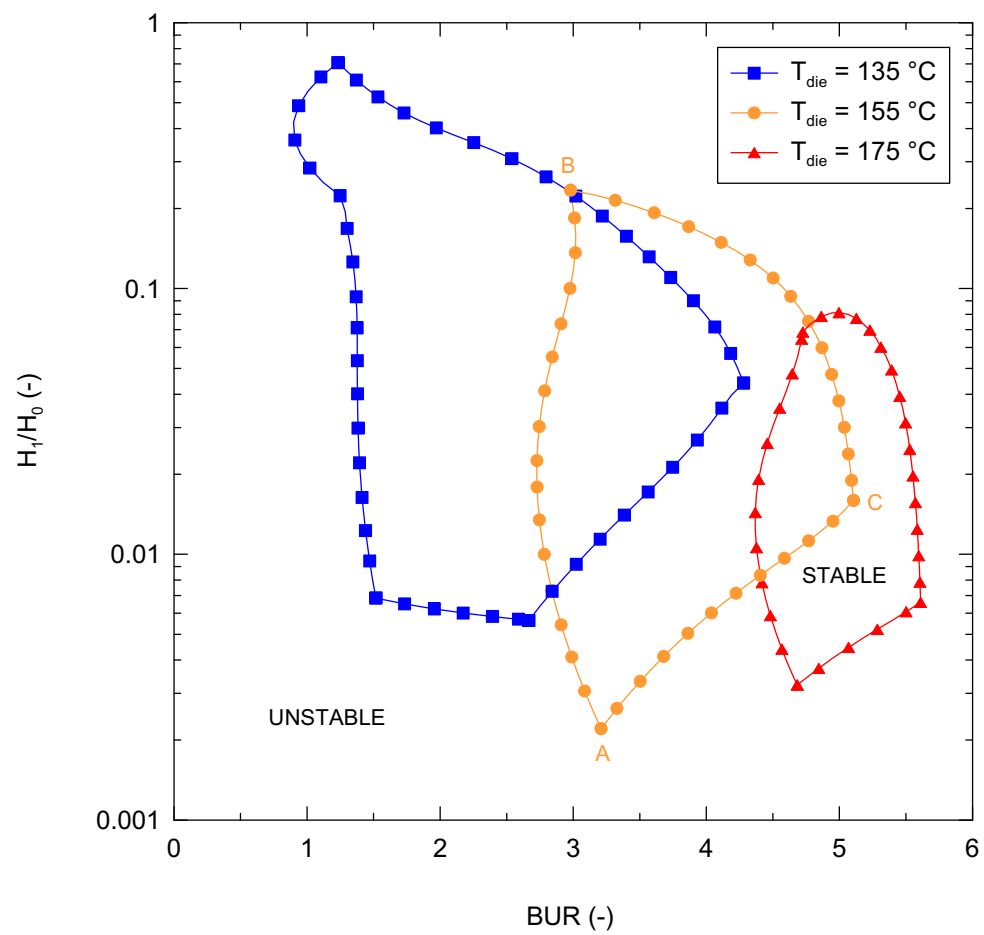

FIGURE 11. Predicted film blowing stability window shape for different level of the melt/die temperature.

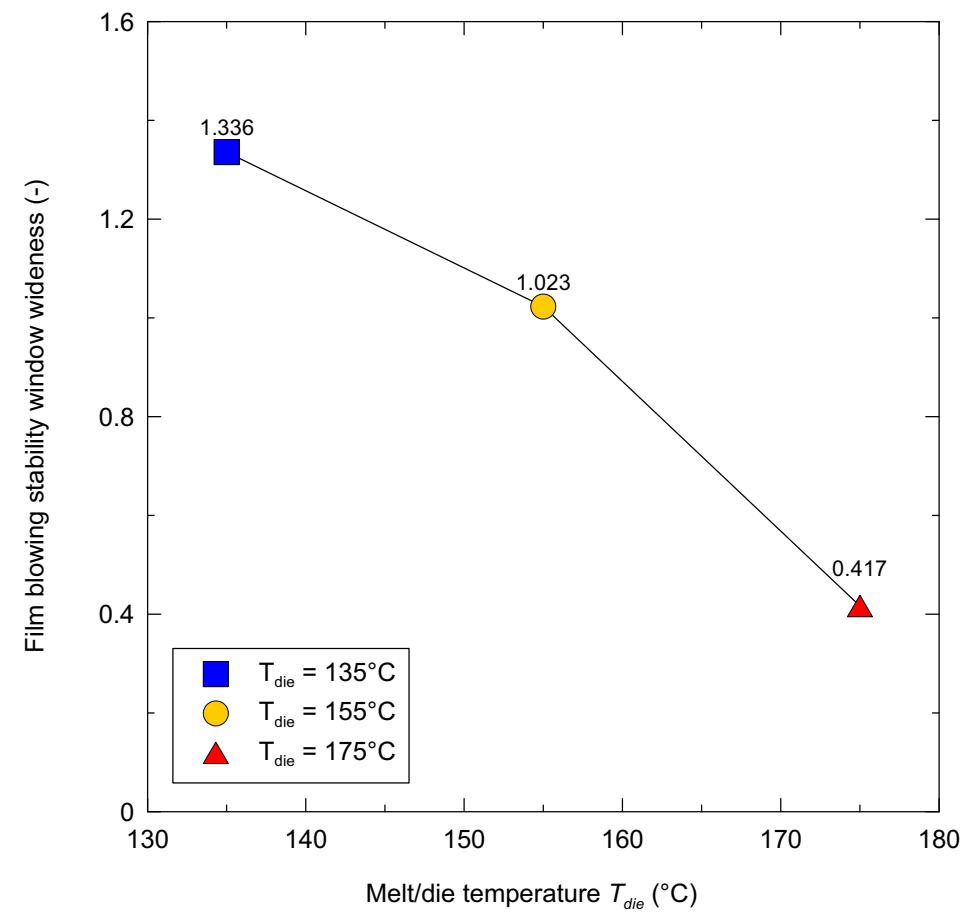

FIGURE 12. Predicted film blowing stability window wideness for different level of the melt/die temperature. 


\section{CONCLUSION}

In this work, a stable numerical scheme for the non-isothermal film blowing stability analysis, considering the variational principle based film blowing model and

non-Newtonian behavior of the polymer, has been utilized to investigate the effect of flow activation energy, heat transfer coefficient, mass flow rate and melt temperature on the film blowing stability. It has been found that the model predictions are in good agreement with the corresponding experimental data taken from the open literature which suggests that it can be considered as the useful tool for the film blowing process optimization.

\section{ACKNOWLEDGMENTS}

The authors wish to acknowledge Grant Agency of the Czech Republic (grant No. P108/10/1325) and the Ministry of Education, Youth and Sports of the Czech Republic (MSM 7088352101) for the financial support. This article was written with support of Operational Program Research and Development for Innovations co-funded by the European Regional Development Fund (ERDF) and national budget of Czech Republic, within the framework of project Centre of Polymer Systems (reg. number: CZ.1.05/2.1.00/03.0111).

\section{REFERENCES}

1. D. V. Rosato, Extruding Plastics - A Practical Processing Handbook, London: Chapman \& Hall, 2005, pp. 305-348.

2. K. Cantor, Blown Film Extrusion, Munich: Cark Hanser Verlag, 2006, pp. 1-13.

3. T. I. Butler, Film extrusion Manual: Process, materials, properties. Atlanta: Tappi press, 2005.

4. P. Waller, What to do when the bubble won't behave, Plastic Technology, (December 2002), pp. 36-37.

5. T. I. Butler, SPE ANTEC Tech. Papers 1120-1129 (2000).

6. J. R. A. Pearson and C. J. S. Petrie, J. Fluid. Mech. 40, 1-19 (1970).

7. J. R. A. Pearson and C. J. S. Petrie, J. Fluid. Mech. 42, 609-625 (1970).

8. J. R. A. Pearson and C. J. S. Petrie, Plast. Polym. 38, 85-94 (1970).

9. J.J. Cain, M.M. Denn, Polym. Eng. Sci. 28, 1527-1541 (1988).

10. M. Zatloukal and J. Vlcek, J. Non-Newtonian Fluid Mech. 123, 201-213 (2004).

11. R. Kolarik, M. Zatloukal, J. Appl. Polym. Sci. (2011). DOI 10.1002/app.34392.

12. A. K Doufas and A. J. McHugh, J. Rheol. 45, 1085-1104 (2001).

13. R. Kolarik, M. Zatloukal, AIP Conference Proceedings 1152, 251-269 (2009).

14. M. Zatloukal, J. Non-Newtonian Fluid Mech. 165, 592-595 (2010).

15. P. P. Tas, "Film blowing from polymer to product", Ph.D. Thesis, Technische Universiteit Eindhoven, 1994.

16. S. Kim, Y. L. Fang, P. G. Lafleur and P. J. Carreau, Polym. Eng. Sci. 44, 283-302 (2004). 
Copyright of AIP Conference Proceedings is the property of American Institute of Physics and its content may not be copied or emailed to multiple sites or posted to a listserv without the copyright holder's express written permission. However, users may print, download, or email articles for individual use. 